九州大学学術情報リポジトリ

Kyushu University Institutional Repository

\title{
GUIDELINES FOR PRACTICAL NAVIGATION SYSTEMS BASED ON WIDE-FIELD-INTEGRATION OF OPTIC FLOW
}

Kobayashi, Naoto

Department of Aeronautics and Astronautics, Kyushu University

Bando, Mai

Department of Aeronautics and Astronautics, Kyushu University

HOKAMOTO, Shinj i

Department of Aeronautics and Astronautics, Kyushu University

Kubo, Daisuke

Japan Aerospace Exploration Agency

http://hdl. handle. net/2324/4479598

出版情報: Asian Journal of Control, 2020-07-01. Chinese Automatic Control Society バージョン：

権利関係 : 


\title{
GUIDELINES FOR PRACTICAL NAVIGATION SYSTEMS BASED ON WIDE-FIELD-INTEGRATION OF OPTIC FLOW
}

\author{
Naoto Kobayashi, Mai Bando, Shinji Hokamoto and Daisuke Kubo
}

\begin{abstract}
This paper shows some guidelines to improve the estimation accuracy of a navigation system, which is based on Wide-Field-Integration (WFI) of optic flow. Optic flow is a vector field of relative velocities obtained by photoreceptors in image sensors, and WFI of optic flow enables motion estimation robust by integrating wide range of optic flow. Since the system has several attractive features (small size, light weight, and low computation), it is applicable to an autonomous control system of micro air vehicles. However, due to some restrictions of real systems, WFI of optic flow theory should be applied while considering sensor arrangement for better estimation. In this paper, first an adequate number and adequate optical-axes of image sensors are investigated. Then, a new estimation system combining a gyro sensor with WFI of optic flow is discussed by numerical simulations. Finally, the essential effect for the number of cameras are verified in experiments.
\end{abstract}

KeyWords: State estimation, optic flow, insect vision, micro air vehicle, WideField-Integration, camera arrangement

\section{INTRODUCTION}

Recently the demands of autonomous navigation systems for Micro Air Vehicles (MAVs) has significantly increased, both in military applications and in commercial environments. However, most of current autonomous control systems for MAVs require an external signal from Global Positioning System (GPS), which gives the vehicle's position in high rates. Thus, autonomous flight in GPS-denied environment (such as inside of buildings) is still an open problem [1, 2, 3]. Major challenges for MAVs are the limitations for size, payload, performance of onboard computer, and battery life. A laser sensor is one possibility, but its weight and energy consumption are serious drawback for MAVs. On the other hand, although a stereovision system can be composed of lightweight cameras, it requires much computational load.

\footnotetext{
Manuscript received September, 2019.

Naoto Kobayashi was a Ph.D candidate with the Department of Aeronautics and Astronautics, Kyushu University, 744 Motooka, Nishi-ku, Fukuoka 819-0395, Japan.

M. Bando with the Associate Professor of Department of Aeronautics and Astronautics, Kyushu University, Japan. Email: mbando@aero.kyushu-u.ac.jp

S. Hokamoto with the Professor of Department of Aeronautics and Astronautics, Kyushu University, Japan. Email: hokamoto@aero.kyushu-u.ac.jp

D. Kubo is with the Researcher of Japan Aerospace Exploration Agency, Japan. Email: kubo.daisuke@jaxa.jp
}

This study applies Wide-Field-Integration (WFI) of optic flow to an autonomous navigation system for MAVs. That is a motion estimation method inspired by a biological research on the visual processing system of flying insects' compound eyes [4, 5]. Optic flow is the vector field of relative velocities to surrounding objects, and it is obtained by photoreceptors in image sensors [6, 7]. This method has attractive features for autonomous navigation systems of MAVs: small-sized and lightweighted cameras are applicable, and its computational load is low [8, 9, 10, 11, 12, 13].

However, WFI of optic flow is hardly applied to practical flight systems because of some gaps between the theory and practical systems. Most influential assumption in the theory is that optic flow is obtained in the whole direction on a spherical image surface. However, the field of view of practical image sensors is restricted. To provide whole directional measurement, applying a whole directional camera leads large distortion on its image surface, and combining several image sensors induces a risk of multiple-counting or vacant area of optic flow and increases computational load.

This paper shows some guidelines to design a practical system with better estimation accuracy by using a few image sensors. In constructing a such navigation system, the number of sensors and their optical-axis directions are key design parameters. Our past study [14] has dealt with one camera case and discussed the 


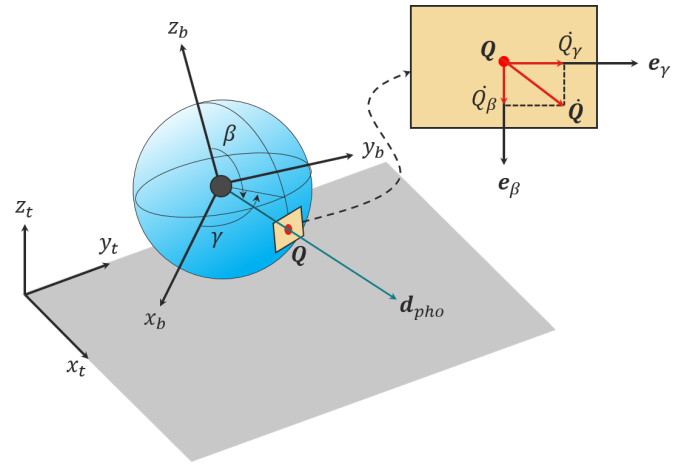

Fig. 1: Geometry for optic flow on spherical imaging surface.

effect of its optical-axis direction on estimation accuracy. Moreover, the study also pointed out that one image sensor frequently results in wrong estimation, because some optic flow patterns observed in a limited field of view may be quite resemble for different motions. This paper discusses the estimation accuracy of systems equipped with multiple sensors, and moreover proposes a new estimation system combining WFI of optic flow and a gyro sensor (WFI-gyro system). This is based on that flying vehicles usually equip with a gyro sensor, and the angular velocities are obtained from it. Thus for a WFIgyro system, the number of estimation variables can be reduced, and this could improve the estimation accuracy. Finally, some guidelines for better estimation accuracy derived from numerical simulations are experimentally verified with practical optic flow sensors mounted on a flying vehicle.

\section{THEORY OF WIDE-FIELD-INTEGRATION OF OPTIC FLOW}

\subsection{Optic flow model}

Optic flow is the vector field of relative velocities obtained by photoreceptors in an image sensor. Describe the total sensor area with a spherical imaging surface $S^{2}$, which is defined around the mass center of a vehicle (Fig. 1). Each point on the spherical surface is described with two angles: azimuth angle $\gamma$ and elevation angle $\beta$. Then the line-of-sight vector of a point on the spherical image surface is written as follows:

$$
\boldsymbol{Q}=\left[\begin{array}{lll}
\sin \beta \cos \gamma & \sin \beta \sin \gamma & \cos \beta
\end{array}\right]^{T}
$$

The fiducial points on the terrain are assumed stationary relative to the terrain-fixed reference frame. The motion parallax vector $\dot{Q}$ is the time derivative of $\boldsymbol{Q}$ on the spherical image surface, which is generated by a combination of the vehicle's translational and rotational motions. Let $\boldsymbol{v}=\left[\begin{array}{ll}u & w\end{array}\right]^{T}$ be the translational velocity vector and $\boldsymbol{\omega}=[p q r]^{T}$ be the angular velocity vector. Then optic flow can be expressed as

$$
\dot{\boldsymbol{Q}}=-\boldsymbol{\omega} \times \boldsymbol{Q}-\mu(\boldsymbol{v}-\langle\boldsymbol{v}, \boldsymbol{Q}\rangle \boldsymbol{Q})
$$

where $\langle *, *\rangle$ denotes the inner product, and $\mu$ is the nearness function defined by the inverse of the distance from the vehicle's mass center to a point on the terrain along the line-of-sight vector. The optic flow $\dot{\boldsymbol{Q}}\left(=\dot{Q}_{\gamma} \boldsymbol{e}_{\gamma}+\dot{Q}_{\beta} \boldsymbol{e}_{\beta}\right)$ has two components along the azimuth and elevation directions, where $\boldsymbol{e}_{\gamma}$ and $\boldsymbol{e}_{\beta}$ are unit vectors on a local tangential plane as shown in Fig. 1.

$$
\begin{aligned}
& \dot{Q}_{\gamma}=p c \beta c \gamma+q c \beta s \gamma-r s \beta+\mu(u s \gamma-v c \gamma) \\
& \dot{Q}_{\beta}=p s \gamma-q c \gamma-\mu(u c \beta c \gamma+v c \beta s \gamma-w s \beta)
\end{aligned}
$$

where $c(*)$ and $s(*)$ mean $\cos (*)$ and $\sin (*)$, respectively. Note that optic flow is obtained in two dimensional image surface and the depth component is zero.

\subsection{Wide-Field-Integration process}

Motion estimation from a few optic flow vectors has been studied over 50 years. However, the estimation accuracy is not satisfactory, because optic flow is a function of unknown vehicle's motion and the nearness function $\mu$, which means unknown parameters of terrain roughness are included implicitly. Contrary to that, WFI of optic flow process utilizes optic flow over a wide area and virtually assumes a flat plane for motion estimation relative to the plane. When the terrain is modeled as a flat, infinite, and horizontal plane, the nearness function $\mu$ is given by

$$
\mu=\frac{-s \beta c \gamma s \theta+s \beta s \gamma s \phi c \theta+c \beta c \phi c \theta}{z}
$$

where $\phi$ and $\theta$ are the roll and pitch angles of the vehicle expressed in 3-2-1 Euler angles, and $z$ is the altitude. It should be noted that this flat surface assumption does not restrict environments which WFI of optic flow process is applicable. Even for unknown real rough terrains, a virtual flat surface can be defined as an averaged plane for a rough terrain.

For the integration of optic flow, a "sensitivity" function is necessary. When a sensitivity function $\boldsymbol{F}_{j}\left(=F_{j}^{\gamma} \boldsymbol{e}_{\gamma}+F_{j}^{\beta} \boldsymbol{e}_{\beta}\right)$ is defined as a square-integrable function, the $j$ th WFI output is defined as

$$
\begin{aligned}
y_{j} & =\left\langle\dot{\boldsymbol{Q}}, \boldsymbol{F}_{j}\right\rangle=\int_{S^{2}} \dot{\boldsymbol{Q}} \cdot \boldsymbol{F}_{j} d \Omega \\
& =\int_{S^{2}}\left(\dot{Q}_{\gamma} F_{j}^{\gamma}+\dot{Q}_{\beta} F_{j}^{\beta}\right) d \Omega
\end{aligned}
$$


where $d \Omega=\sin \beta d \beta d \gamma$ is a solid angle on the spherical image surface. As the sensitivity functions, Fourier series are typically used for two-dimensional cases. Whereas for the estimation of three-dimensional motion, this study uses spherical harmonics as $\boldsymbol{F}_{j}$. For the 1st$M$ th sensitivity functions, one can construct an $M \times 1$ measurement vector for WFI of outputs as follows:

$$
\boldsymbol{y}=\left[\begin{array}{lll}
y_{1} & \cdots & y_{M}
\end{array}\right]^{T}
$$

In general, Eqs. (6) and (7) are used in a standard WFI of optic flow process. However, to separately evaluate two optic flow components and improve the estimation accuracy, our past study[15] has proposed to use the following equations.

$$
\boldsymbol{y}=\int_{S^{2}} \dot{\boldsymbol{Q}} \cdot\left[\begin{array}{cc}
\boldsymbol{F}^{\gamma} & \mathbf{0} \\
\mathbf{0} & \boldsymbol{F}^{\beta}
\end{array}\right] d \Omega=\left[\begin{array}{l}
\boldsymbol{y}^{\gamma} \\
\boldsymbol{y}^{\beta}
\end{array}\right]
$$

where

$$
\begin{aligned}
& \boldsymbol{y}^{\gamma}=\left[\begin{array}{lll}
\int_{S^{2}} \dot{Q}_{\gamma} F_{1}^{\gamma} d \Omega & \cdots & \int_{S^{2}} \dot{Q}_{\gamma} F_{M}^{\gamma} d \Omega
\end{array}\right]^{T} \\
& \boldsymbol{y}^{\beta}=\left[\begin{array}{lll}
\int_{S^{2}} \dot{Q}_{\beta} F_{1}^{\beta} d \Omega & \cdots & \int_{S^{2}} \dot{Q}_{\beta} F_{M}^{\beta} d \Omega
\end{array}\right]^{T}
\end{aligned}
$$

Thus WFI output, $\boldsymbol{y}=\left[y_{1}^{\gamma} \cdots y_{M}^{\gamma} y_{1}^{\beta} \cdots y_{M}^{\beta}\right]^{T}$, is a $2 M \times 1$ measurement vector.

Furthermore, in a standard theory shown in [4, 9. [10], optic flow $\dot{Q}$ is regarded as a continuous function. However, in a real system, the optic flow data is obtained by photoreceptors on an image sensor at discretized points. Thus, for simple calculations, the integration process of WFI output is approximated by Riemann sums as follows:

$$
\boldsymbol{y}=\left[\begin{array}{c}
\sum_{k=1}^{N} \dot{Q}_{\gamma}\left(\gamma_{k}, \beta_{k}\right) F_{1}^{\gamma}\left(\gamma_{k}, \beta_{k}\right) \Delta \Omega \\
\vdots \\
\sum_{k=1}^{N} \dot{Q}_{\gamma}\left(\gamma_{k}, \beta_{k}\right) F_{M}^{\gamma}\left(\gamma_{k}, \beta_{k}\right) \Delta \Omega \\
\sum_{k=1}^{N} \dot{Q}_{\beta}\left(\gamma_{k}, \beta_{k}\right) F_{1}^{\beta}\left(\gamma_{k}, \beta_{k}\right) \Delta \Omega \\
\vdots \\
\sum_{k=1}^{N} \dot{Q}_{\beta}\left(\gamma_{k}, \beta_{k}\right) F_{M}^{\beta}\left(\gamma_{k}, \beta_{k}\right) \Delta \Omega
\end{array}\right]
$$

where $N$ is the total number of photoreceptors on the imaging surface. Note, our past study [16] shows that this Riemann sum approximation does not lower the estimation accuracy.

\subsection{Estimation of state vector}

Vehicle's motion is defined by 12 variables for its position, attitude, and their derivatives. Denote $x, y, z, \phi, \theta, \psi$ as the position/attitude variables and $u, v, w, p, q, r$ as the translational/rotational velocities, all of which are defined with respect to the terrain-fixed frame. However, from the flat, infinite and horizontal ground assumption, three components $(x, y$ and $\psi)$ are meaningless and deleted from the estimation variables. Thus the state vector is defined as

$$
\boldsymbol{x}_{o}=\left[\begin{array}{lllllllll}
z & u & v & w & \phi & \theta & p & q & r
\end{array}\right]^{T}
$$

Furthermore, Eqs. (3) and (4) indicate that the optic flow components are liner with respect to the vehicle's translational and angular velocities. Thus, when the state variables is defined as

$$
\boldsymbol{x}=\left[\begin{array}{llllll}
u & v & w & p & q & r
\end{array}\right]^{T}
$$

the WFI output $\boldsymbol{y}$ can be expressed in a linear form as

$$
\boldsymbol{y}=\boldsymbol{C}(\phi, \theta, z) \boldsymbol{x}
$$

where

$$
\boldsymbol{C}(\phi, \theta, z)=
$$

$$
\sum_{k=1}^{N}\left[\begin{array}{ccc}
\mu_{k} s \gamma_{k} F_{1}^{\gamma} & -\mu_{k} c \gamma_{k} F_{1}^{\gamma} & 0 \\
\vdots & \vdots & \vdots \\
\mu_{k} s \gamma_{k} F_{M}^{\gamma} & -\mu_{k} c \gamma_{k} F_{M}^{\gamma} & 0 \\
-\mu_{k} c \beta_{k} c \gamma_{k} F_{1}^{\beta} & -\mu_{k} c \beta_{k} s \gamma_{k} F_{1}^{\beta} & \mu_{k} s \beta_{k} F_{1}^{\beta} \\
\vdots & \vdots & \vdots \\
-\mu_{k} c \beta_{k} c \gamma_{k} F_{M}^{\beta} & -\mu_{k} c \beta_{k} s \gamma_{k} F_{M}^{\beta} & \mu_{k} s \beta_{k} F_{M}^{\beta} \\
c \beta_{k} c \gamma_{k} F_{1}^{\gamma} & c \beta_{k} s \gamma_{k} F_{1}^{\gamma} & -s \beta_{k} F_{1}^{\gamma} \\
\vdots & \vdots & \vdots \\
c \beta_{k} c \gamma_{k} F_{M}^{\gamma} & c \beta_{k} s \gamma_{k} F_{M}^{\gamma} & -s \beta_{k} F_{M}^{\gamma} \\
s \gamma_{k} F_{1}^{\beta} & -c \gamma_{k} F_{1}^{\beta} & 0 \\
\vdots & \vdots & \vdots \\
s \gamma_{k} F_{M}^{\beta} & -c \gamma_{k} F_{M}^{\beta} & 0
\end{array}\right] \Delta \Omega_{k}
$$

$C$ is an observation matrix of $2 M \times 6$, whose components include three variables $(\phi, \theta, z)$. Thus, when these three variables are measured, the WFI sensor output vector $y$ and the state variables $\boldsymbol{x}$ are linear relations. (There are researches trying to estimate the position and attitude of a vehicle from onboard optical image sensors, e.g., [17, 18, 19]. However, to clearly discuss the effect of the number and directions of image sensors, this paper 
assumes these three variables are measured by other sensors and focuses on the estimation of translational and rotational velocities.)

Then, the state vector $\boldsymbol{x}$ is estimated through the pseudo-inverse matrix of $\boldsymbol{C}$ as follows:

$$
\boldsymbol{x}=\boldsymbol{C}^{\dagger}(\phi, \theta, z) \boldsymbol{y}
$$

where

$$
\boldsymbol{C}^{\dagger}=\left(\boldsymbol{C}^{T} \boldsymbol{C}\right)^{-1} \boldsymbol{C}^{T}
$$

\subsection{Estimation errors}

Let $\boldsymbol{\eta}$ be the error included in the WFI of optic flow output due to the observation noise and the roughness of unknown real terrain. Then estimated state vector $\tilde{\boldsymbol{x}}$ in the WFI of optic flow is written as follows:

$$
\tilde{\boldsymbol{x}}=\boldsymbol{C}^{\dagger}(\boldsymbol{y}+\boldsymbol{\eta})=\boldsymbol{x}+\boldsymbol{C}^{\dagger} \boldsymbol{\eta}
$$

where the term of $\boldsymbol{C}^{\dagger} \boldsymbol{\eta}$ indicates the estimation errors of the motion variables. Note that the errors can be decreased by increasing the number of photoreceptors or by expanding the integrated area, because optic flow noises at photoreceptor positions are canceled each other through the integration process. Furthermore, since the observation matrix $\boldsymbol{C}$ is a function of $(\beta, \gamma)$, the optical axis of an image sensor affects the estimation accuracy of motion variables.

\section{EFFECT OF CAMERAS ON ESTIMATION ACCURACY}

It is known that image sensors with a wide field of view and high resolution are desirable for WFI of optic flow [15]. However, composing such preferable images by several high resolution sensors results in increasing both the total weight of sensor systems and the computational load. Thus, at the beginning for discussions of estimation accuracy, this section examines in numerical simulations how estimation accuracy changes according to the number of cameras. The results give a guideline to decide the number of image sensors in design phase, considering realistic restrictions for cameras.

As an example, flight condition of a vehicle is set as shown in Table 1. Let us explain that optic flow is essentially obtained by a moving camera. However, when white noises are added to ideal optic flow to express roughness of terrain surface and measuring noises, optic flow is obtained by a stationary camera. Furthermore, from our several preliminary simulations, motion estimation obtained for a stationary condition has the same features as for moving conditions. Thus as shown in Table 1, a vehicle is assumed to be hovering,
Table 1: Flight conditions of a vehicle.

\begin{tabular}{cc}
\hline altitude $[\mathrm{m}]$ & 1 \\
attitude $[\mathrm{deg}]$ & $\phi=0, \quad \theta=0, \quad \psi=0$ \\
velocity $[\mathrm{m} / \mathrm{s}]$ & $u_{t}=0, \quad v_{t}=0, \quad w_{t}=0$ \\
angular velocity $[\mathrm{deg} / \mathrm{s}]$ & $p=0, \quad q=0, \quad r=0$ \\
simulation time & $0.1 \mathrm{~s}$ interval during $0 \mathrm{~s}$ to $10 \mathrm{~s}$
\end{tabular}

Table 2: Optical axes of the cameras used in simulations.

\begin{tabular}{ccc}
\hline Camera ID & $\beta[\mathrm{deg}]$ & $\gamma[\mathrm{deg}]$ \\
\hline 1 & 135 & 45 \\
2 & 135 & 135 \\
3 & 135 & 225 \\
4 & 135 & 315 \\
\hline
\end{tabular}

and white noises with zero-mean-value and $0.3 \mathrm{rad} / \mathrm{s}$ standard-deviation are added to ideal optic flow. From optic flow patterns obtained in 0.1 second interval, the vehicle's motion variables are estimated. Note that since the state variables are estimated around their true values by applying WFI of optic flow process, in the simulations below only the standard deviations of the estimated values are shown and they indicate the estimation accuracy.

As a practical image sensor, the field of view is set as 30 and 40 degrees in its elevation and azimuth directions, respectively. In image surface, optic flow is assumed to be obtained at every 1-degree in both directions. In addition, the optical-axes of image sensors are directed as shown in Table 2: from the results in [14], the elevation direction is fixed to 135 degrees for better estimation. The camera ID indicates the cameras used in the simulations: for example, if the number of cameras is one, the camera of ID 1 is used, and the cameras of ID 1, 2, and 3 are used for three cameras.

Figure 2 compares the standard deviations of estimated errors when the number of cameras is changed from one to four. Each color of the bars indicates the number of cameras. The result shows that the standard deviations of estimated errors are reduced as the number of cameras increases. From this simulation, roughly three are adequate for the number of cameras from considering benefits and disadvantages, because the improvement for adding fourth camera is limited.

\section{EFFECT OF CAMERAS DIRECTIONS}

Effects on estimation accuracy of the optical-axis in one camera case have already been investigated in our previous work [14]. It also indicates that when the field of view is limited, one image sensor frequently encounters difficulty to distinguish optic flow patterns of different motions. This section discusses adequate optical axes of multiple cameras for WFI of optic flow. The flight condition of a vehicle is the same as Table 1, and the 


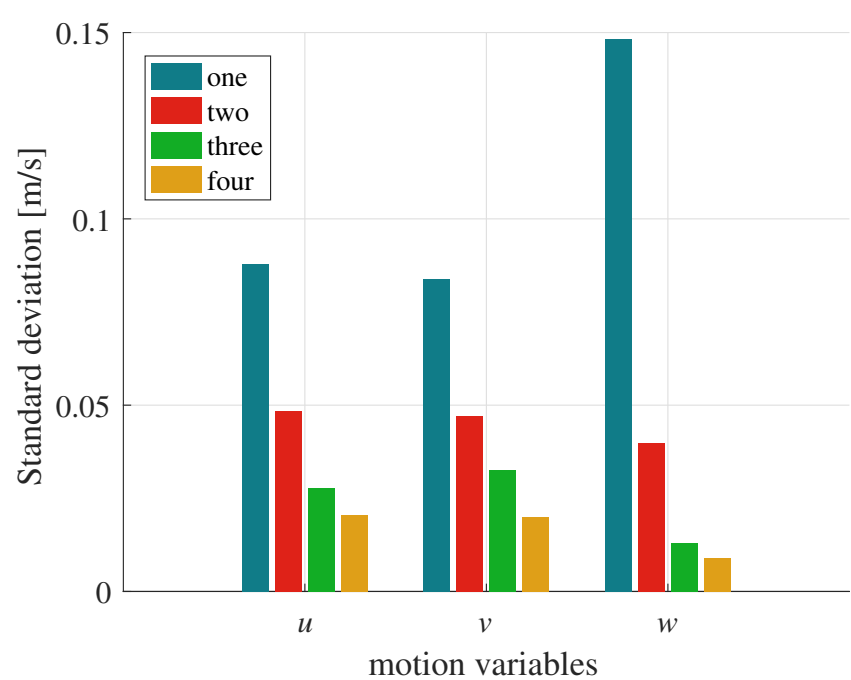

(a) Standard deviations of estimated errors in translational velocities

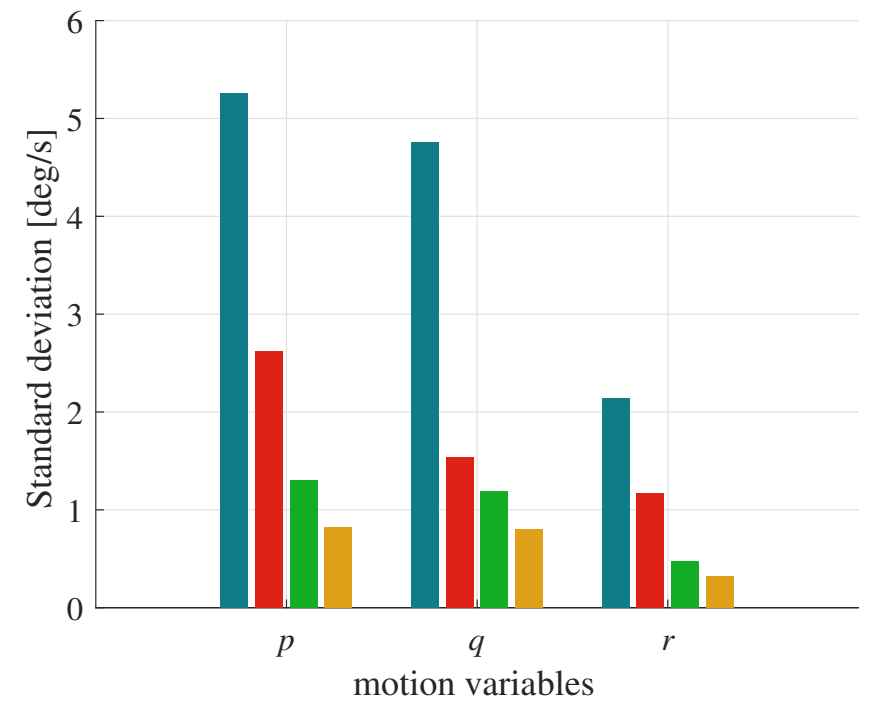

(b) Standard deviations of estimated errors in angular velocities

Fig. 2: Comparison of the standard deviations of estimated errors for each number of cameras.

specifications of cameras and sensor noises are also the same as the previous simulation.

\subsection{Single camera}

First, the effect of an optical axis direction for one camera is reexamined for reference: the effect of the elevation angle is examined in Case 1, and that of the azimuth angle is examined in Case 2. In Case 1, the azimuth direction of the camera's optical-axis is fixed to 0 degree, and the elevation direction is changed with 15-degree interval from 105 to 165 degrees (90-degree means horizontal direction in this flight condition). While in Case 2, the azimuth direction is changed with 15-degree interval from 0 to 345 degrees, while the elevation direction is fixed to 135 degrees.

Figures 3 and 4 show the standard deviations of the estimated errors in Case 1 and Case 2, respectively. Figure 3 indicates that as the elevation direction of the optical axis approaches to a horizontal direction $(\beta=90 \mathrm{deg})$, the estimation accuracy of almost all motion variables becomes better. However, since optic flow cannot be obtained in its upper hemisphere, this result implies that the optical-axes of cameras should be decided considering the field of view of cameras and vehicle's expected attitude change. Figure 4 indicates that estimation accuracies of each motion variables become periodic for the azimuth angle $\gamma$. This tendency comes from the symmetry of the spherical image surface. In addition, the result implies that, for translational velocities, better estimation accuracy is obtained from a camera facing to the moving direction: $\gamma=0 \mathrm{deg}$ or $180 \mathrm{deg}$ for $u$, and $\gamma=90 \mathrm{deg}$ or $270 \mathrm{deg}$ for $v$. Thus, the result implies that the azimuth direction of camera's optical-axis should be decided according to the vehicle's expected moving direction. For example, if the vehicle is a fixed-wing aircraft, since its moving direction is fixed, the optical axis should be faced to the moving direction. On the other hand, if the vehicle is a multirotor vehicle which moves to any direction, the optical axis should be decided such that the lowest estimation accuracy is allowable for every directional movement.

\subsection{Multiple cameras}

Based on the results in Sec. 4.1, this subsection focuses on how to arrange the azimuth directions of multiple cameras. In below, a vehicle is assumed to be a multirotor vehicle, and the camera directions should be arranged so that the lowest estimation accuracy becomes better.

\subsubsection{Two cameras}

The effect of the azimuth directions of two cameras is examined in Case 3. In this case, the elevation directions of optical-axes of two cameras are fixed at 135 degrees. Furthermore, the azimuth direction of the optical-axis of one camera is fixed at 0 degree, and that of another camera is changed with 15-degree interval from 30 to 330 degrees.

Figure 5 shows the standard deviations of the estimated errors in Case 3. The result indicates that the second camera should be faced to around $\gamma=60 \sim 90$ or $\gamma=270 \sim 300$ degree, in order to reduce the worst estimation error. Considering this result and the symmetry of the spherical image surface, two cameras should be arranged such that their optical-axes are roughly orthogonal to each other. This guideline is consistent with Fig. 4; when a camera is fixed at $\gamma=0$ degree, the estimation accuracies of $u, q$ are good and those of $v, p$ are poor. Thus, to improve the estimation accuracies of $v, p$, the second camera should be mounted around $\gamma=90$ degree. 


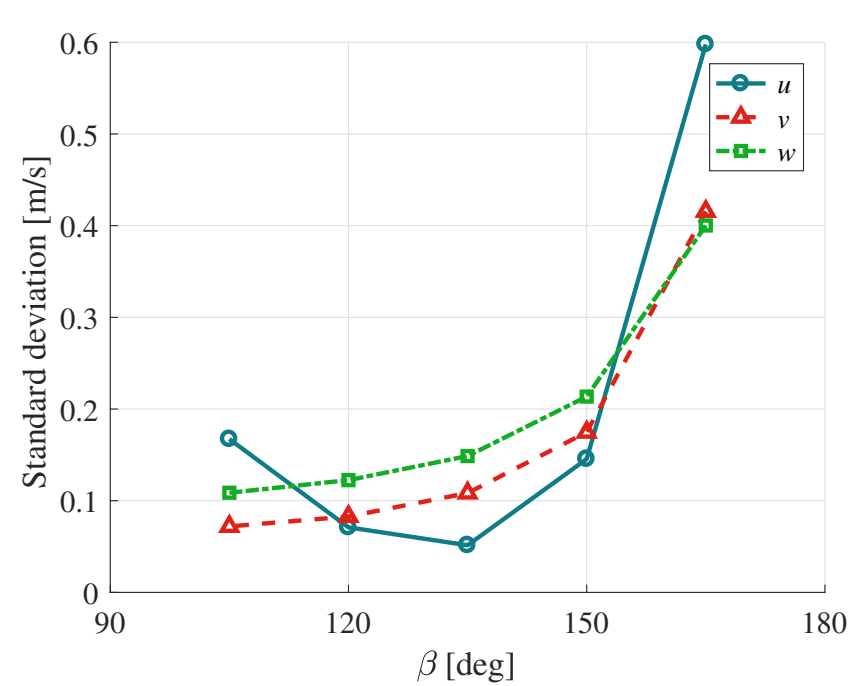

(a) Standard deviations of estimated errors in translational velocities

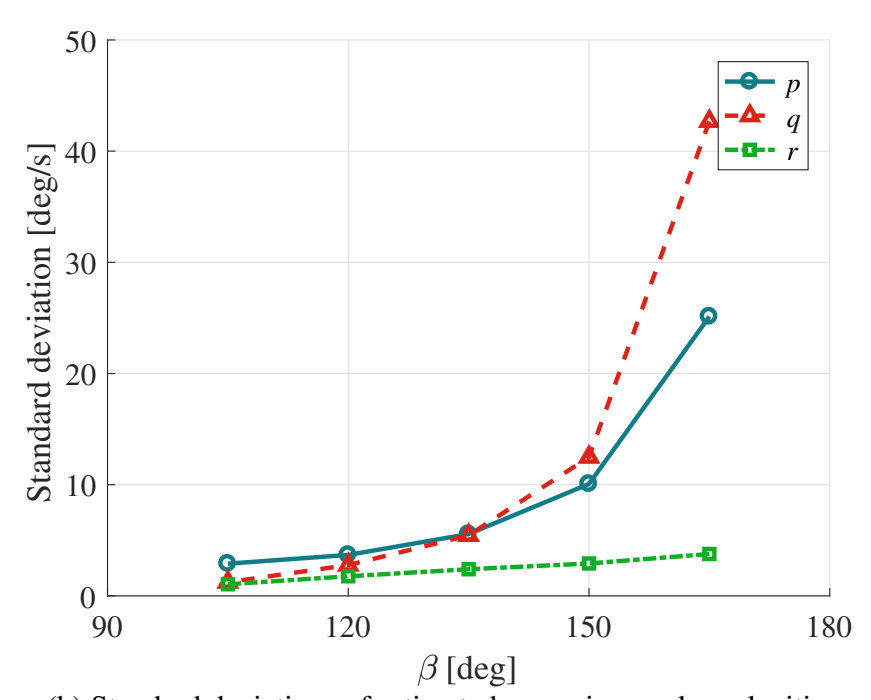

(b) Standard deviations of estimated errors in angular velocities

Fig. 3: Standard deviations of estimated errors in Case 1.

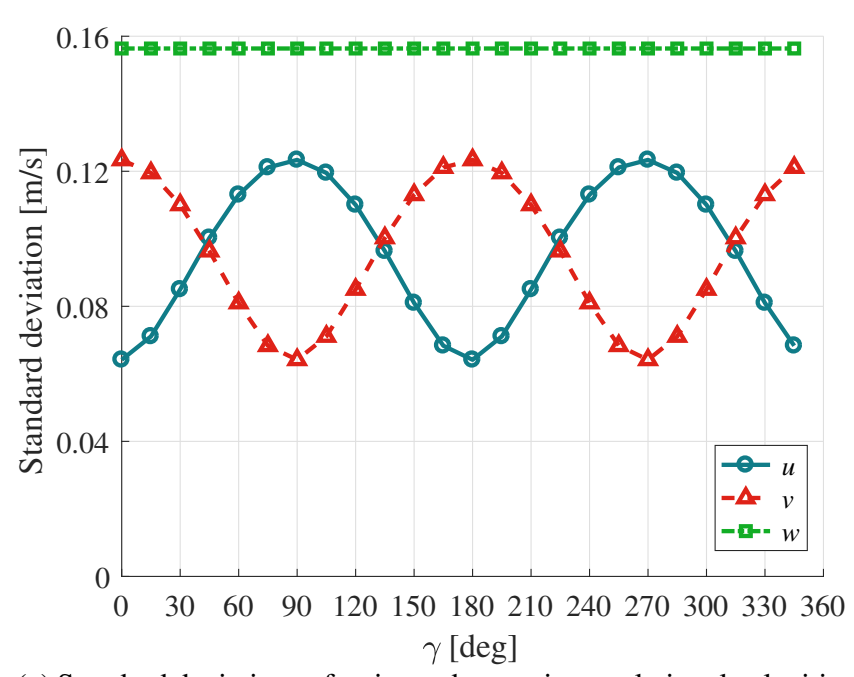

(a) Standard deviations of estimated errors in translational velocities

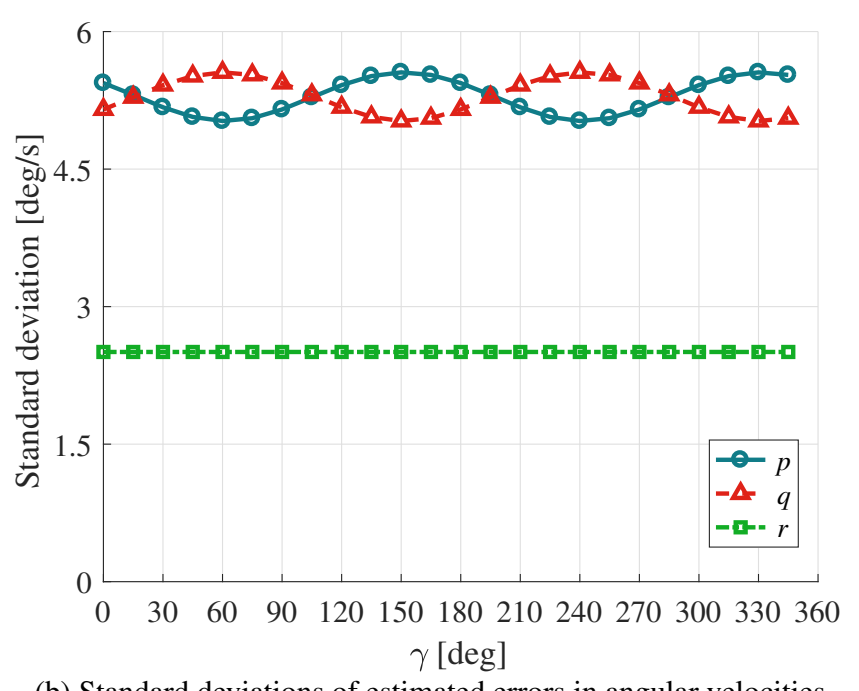

(b) Standard deviations of estimated errors in angular velocities

Fig. 4: Standard deviations of estimated errors in Case 2.

\subsubsection{Three cameras}

Finally, the effect of azimuth angles for three cameras are examined in Case 4. To simplify the discussion, two cameras' azimuth angles are fixed at 0 and 90 degrees, and the third camera direction is changed with 15-degree interval from 30 to 330 degrees. Meanwhile, the elevation angles of the all cameras are fixed at 135 degrees.

Figure 6 indicates the standard deviations of the estimated errors in Case 4. The figure shows that for better estimation, the third camera should be faced to around $\gamma=225$ degrees. This implies the following guideline in azimuth directions: when two cameras are already fixed, the azimuth angle of the third camera should be faced to the center direction for the wider angle defined by other two cameras. Note that the estimation accuracies are not periodic for the azimuth angle as shown in Fig. 6 : the third camera facing to $\gamma=45$ degrees gives much poorer estimation result than the third camera facing to $\gamma=225$ degrees. This implies that the estimation accuracy cannot be improved when a newly added camera gives information for near area already measured by other cameras. That is, since optic flow measured in near areas are usually resemble, the third camera cannot give useful new information to distinguish motion variables.

\subsubsection{Guideline for multiple camera directions}

Subsections 4.2.1 and 4.2.2 imply a guideline for better azimuth directions of multi-cameras; i.e., camera directions should be specified by considering the following two points.

- Each camera direction should be separated as much as possible.

- A camera placed with $\gamma=180 \mathrm{deg}$ from one of 


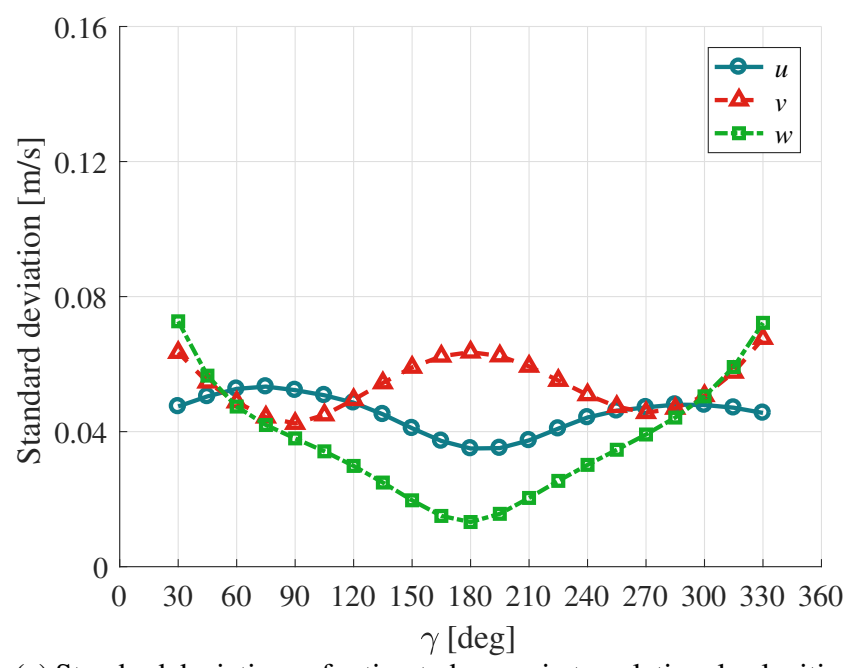

(a) Standard deviations of estimated errors in translational velocities

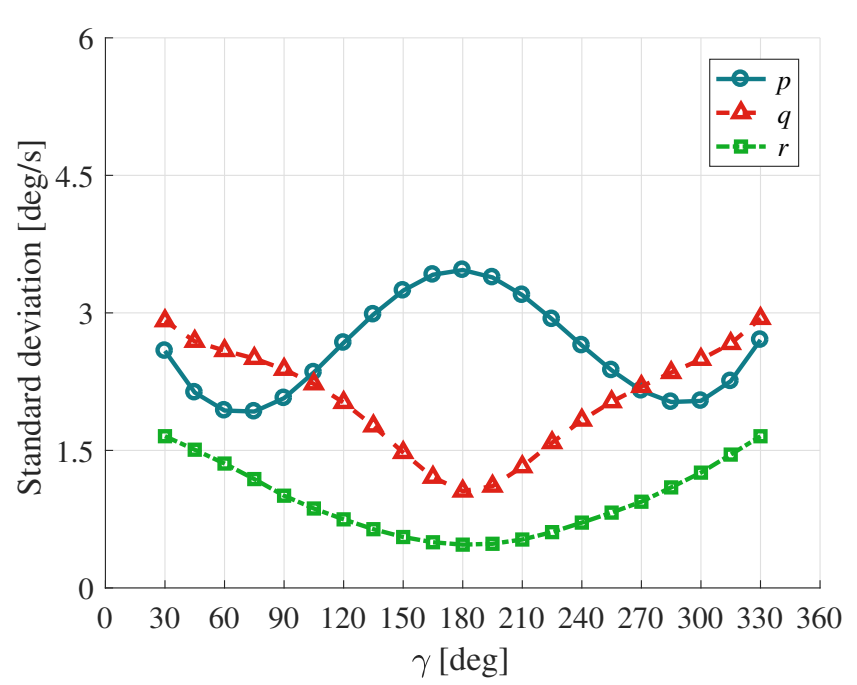

(b) Standard deviations of estimated errors in angular velocities

Fig. 5: Standard deviations of estimated errors in Case 3.

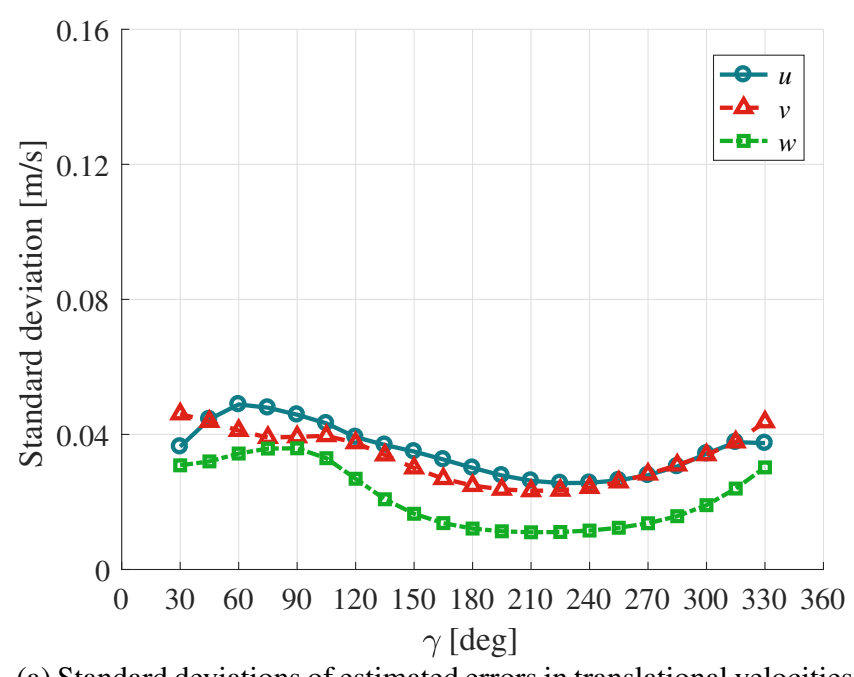

(a) Standard deviations of estimated errors in translational velocities

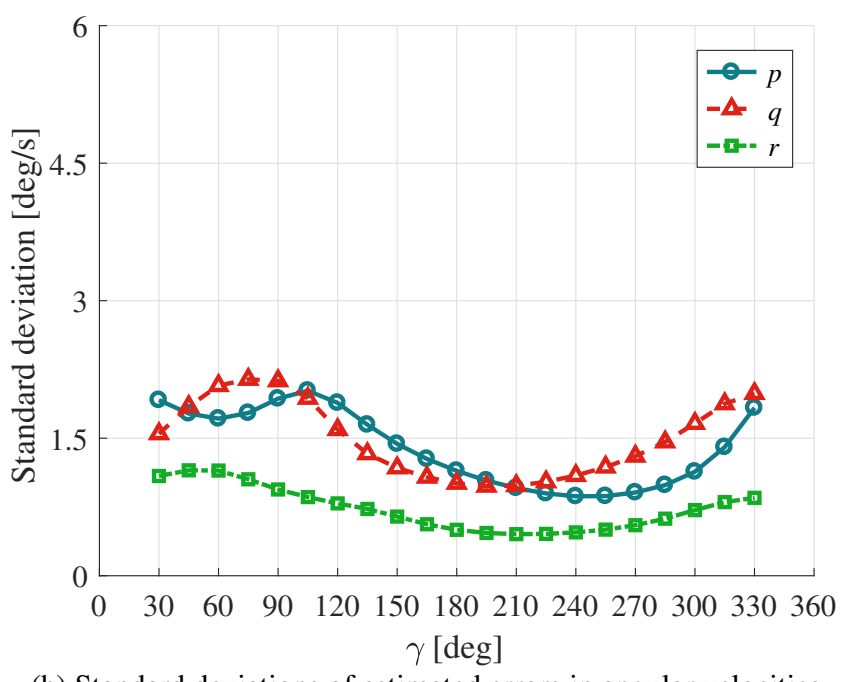

(b) Standard deviations of estimated errors in angular velocities

Fig. 6: Standard deviations of estimated errors in Case 4.

other cameras gives less improvement for variable estimations due to independent optic flow.

From the guideline in above, one proper azimuth directions of three cameras is $\gamma=(0,120,240)$ deg. Indeed, this camera placement improves the estimation accuracy $0 \sim 10 \%$ according to the motion variables compared with the placement of $\gamma=(0,90,225) \mathrm{deg}$ treated in Subsection 4.2.2.

\section{WFI-GYRO SYSTEM}

As described in Sec. 1, flying vehicles usually equip with gyro sensors to measure their angular velocities. This section discusses the effect for estimating only translational velocities from WFI of optic flow.

\subsection{Translational velocity estimation}

For the WFI-gyro system, Eq. (14) is rewritten as follows:

$$
y=A v+B \omega
$$

where $\boldsymbol{A}$ and $\boldsymbol{B}$ are $2 M \times 3$ measurement matrices. When $\boldsymbol{\omega}$ is measured by a gyro sensor, the translational velocity vector $\boldsymbol{v}$ is estimated through the pseudo inverse matrix of $\boldsymbol{A}$ as follows:

$$
\boldsymbol{v}=\boldsymbol{A}^{\dagger}(\boldsymbol{y}-\boldsymbol{B} \boldsymbol{\omega})
$$

In practical systems, sensor noises are included in a gyro sensor output as well as optic flow outputs $\boldsymbol{\eta}$. Let $\delta \boldsymbol{\omega}$ be the gyro sensor noise, then the velocity vector estimated from the WFI-gyro system is written as follows: 


$$
\begin{aligned}
\tilde{\boldsymbol{v}} & =\boldsymbol{A}^{\dagger}\{\boldsymbol{y}+\boldsymbol{\eta}-\boldsymbol{B}(\boldsymbol{\omega}+\delta \boldsymbol{\omega})\} \\
& =\boldsymbol{v}+\boldsymbol{A}^{\dagger}(\boldsymbol{\eta}-\boldsymbol{B} \delta \boldsymbol{\omega})
\end{aligned}
$$

where the term of $\boldsymbol{A}^{\dagger}(\boldsymbol{\eta}-\boldsymbol{B} \delta \boldsymbol{\omega})$ indicates the estimation errors in this WFI-gyro system, and the matrices $\boldsymbol{A}$ and $\boldsymbol{B}$ are the functions of $(\beta, \gamma)$. Comparing Eqs. (18) and (21), it is obvious that the estimation error of WFI-gyro system is different function from WFI optic flow. Thus, the adequate sensor arrangement in WFI-gyro system may be different when WFI of optic flow estimates six motion variables.

\subsection{Numerical simulations}

Note, if accurate angular velocities are obtained from a gyro sensor, better estimation accuracies for translational velocities are expected from a WFI-gyro system. This is based on that the size of $\boldsymbol{A}^{\dagger}$ in Eq. (21) is smaller than $C^{\dagger}$ in Eq. (18) and its components are less contaminated by noises. However, since the output of a real gyro sensor includes noise, the estimation accuracy of a WFIgyro system depends on two noises and this makes the analytical discussion complicated. Thus, instead of comparing the estimation accuracies between a WFI-gyro system and a standard WFI of optic flow system, this paper focuses on an adequate optical-axis direction of a camera in the WFI-gyro system.

Flight conditions in the numerical simulations are the same as the ones shown in Table 1. The directions of the optical axis in each simulation are summarized in Table 3. In Case 5, the effect of the elevation direction of optical axis in the WFI-gyro system is examined, while in Case 6 , the effect of the azimuth direction is investigated. In each case the direction of the optical-axis is changed with 15-degree interval. The specifications of an image sensor are the same as the previous simulations. Sensor noises included in the optic flow is assumed to be white noises, whose mean-value is zero and its standarddeviation is $0.3 \mathrm{rad} / \mathrm{s}$. For a gyro sensor, white noises with zero-mean-value and $5 \mathrm{deg} / \mathrm{s}$-standard-deviations of are used to emphasize the effect of gyro sensor noises.

Figures 7 and 8 show the standard deviations of the estimated translational velocity errors in Cases 5 and 6, respectively. The result of Fig. 7 indicates that the estimation accuracy of the translational velocities become worse as the elevation direction of the optical axis approaches to horizontal direction. Note that this tendency is opposite to Fig. 3 (a) for a standard WFI of optic flow system, and it implies that image sensors should be faced to the vehicle's bottom direction. For the effect of the azimuth direction of a camera in the WFI-gyro system, Fig. 8 indicates an apparent difference is shown in $w$ compared with Fig. 4 (a). Thus, the arrangement of image sensors should be decided according to whether a gyro
Table 3: Direction of optical axis used in simulations for WFI-gyro system.

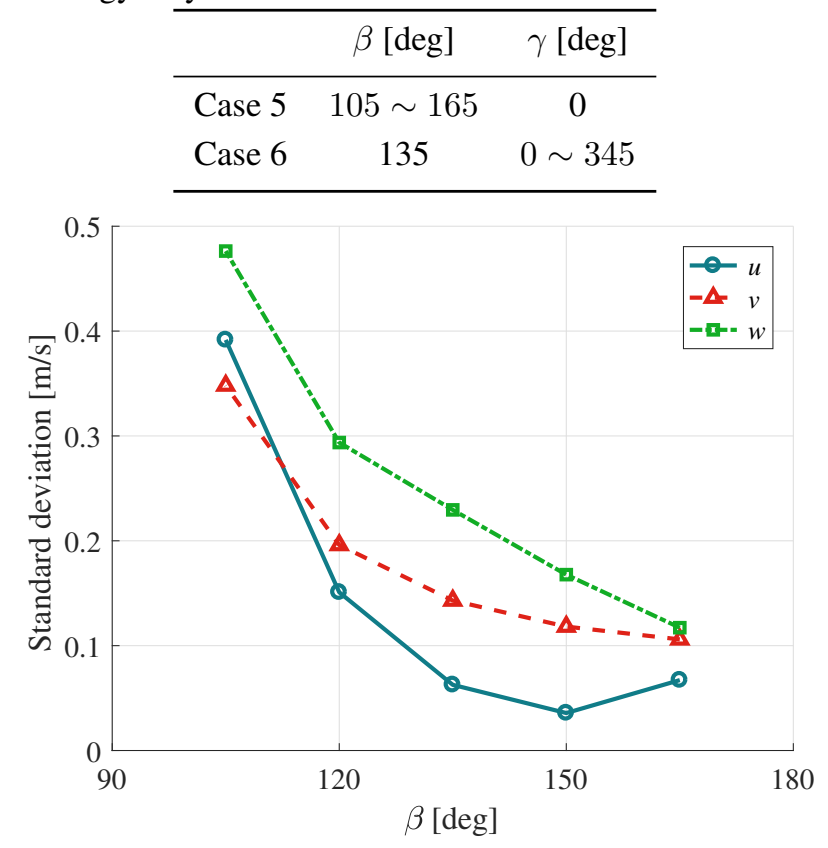

Fig. 7: Standard deviations of estimated errors in Case 5.

sensor is used or not.

\section{EXPERIMENTS}

This section verifies the effects of the number of cameras on the estimation accuracy in experiments. Figure 9 shows the optic flow sensor (OpticalFlow-Z made by ZMP Corp.) used in the experiments. For this sensor, optic flow of $(79 \times 59)$ is obtained at each grid point for the field of view (45.1 deg $\times 34.6 \mathrm{deg}$ ) at every 0.1 second.

One or two optic flow sensors are used for the verification experiments. These optic flow sensors are mounted on a quad-rotor vehicle flying in a room by manual control, and random textures are placed on the floor to make optic flow detection easier. In the sensors, optic flow patterns are obtained with 30 frames/s based on pixel intensities, and the motion variables of the vehicle are estimated by the WFI of optic flow process described in this paper. The direction of one camera is fixed at $(\gamma=0, \beta=135)$ degrees, and another camera is facing to $(\gamma=90, \beta=135)$ degrees direction; these directions are decided from the numerical simulations in the Sec. IV. For the comparison with the estimation results of WFI optic flow, a motion capture system (VICON T-10S) with high resolutions is used to measure the vehicle's motion variables.

Figure 10 shows the time histories of the estimated motion variables. Each line indicates the estimated values with one or two cameras, as well as the results measured by the motion capture system. By assuming the result of the motion capture system is correct, the standard 


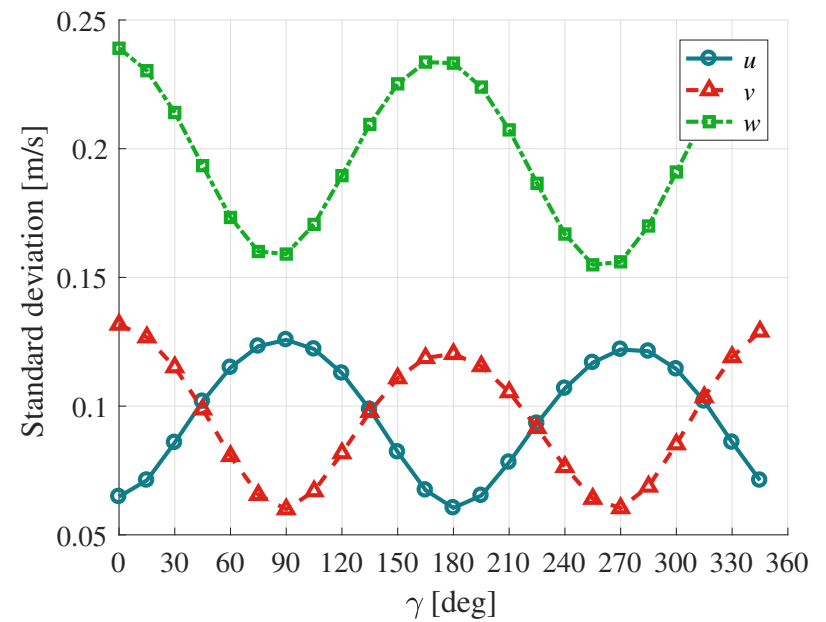

Fig. 8: Standard deviations of estimated errors in Case 6.

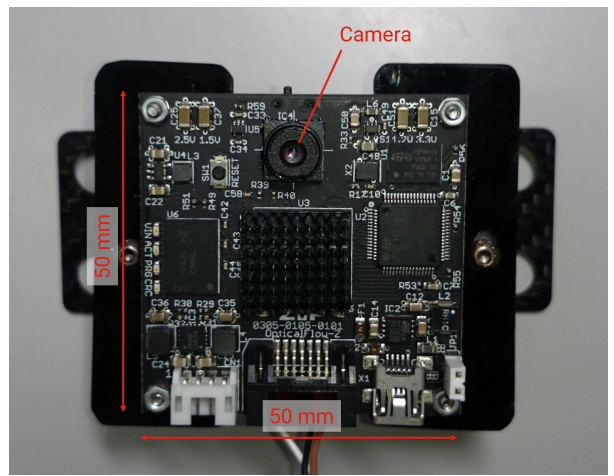

Fig. 9: Optic flow sensor used in experiments

deviations of the errors estimated from the WFI of optic flow sensor are shown in Fig. 11 This result indicates that the standard deviations of the estimated errors can be reduced by increasing the number of cameras from one to two, except for $u$. For the estimation of $u$, the estimation accuracies are almost same; this is because the accuracy of $u$ obtained by one camera is already sufficiently good. Figure 11 is consistent to the results of the numerical simulations.

\section{CONCLUSIONS}

This paper has discussed several design guidelines to improve the estimation accuracy of a system based on Wide-Field-Integration of optic flow, considering the restrictions in real systems. Especially, the adequate number and the optical-axis directions of cameras have been investigated. The results show that the standard deviations of the estimation errors are reduced as the number of cameras is increased. In addition, the directions of image sensors should be arranged considering the vehicle's expected motion. Furthermore, a new system combining a gyro sensor with WFI of optic flow sensors has been proposed in this paper. And for this system, the effect on an optical-axis direction of a camera is opposite to that for a standard WFI of optic flow system. Finally, the effect of camera number has been verified with the experiments, and the obtained result is consistent with the result obtained in the numerical simulations.

The design guidelines obtained in this study make WFI of optic flow practical, and they will enhance the research on autonomous vehicles.

\section{ACKNOWLEDGEMENTS}

This work was supported by Japan Society for the Promotion of Science (JSPS) KAKENHI Grant-in-Aid for JSPS Research Fellow No. 18J11084.

\section{REFERENCES}

1. Shaojie Shen., Nathan Michael. and Vijay Kumar, "Autonomous Multi-Floor Indoor Navigation with a Computationally Constrained MAV" IEEE International Conference on Robotics and Automation (2011).

2. Markus Achtelik., Abraham Bachrach., Ruijie He., Samuel Prentice. and Nicholas Roy, "Autonomous Navigation and Exploration of a Quadrotor Helicopter in GPS-denied Indoor Environments" First Symposium on Indoor Flight. (2009).

3. David Droeschel., Jorg Stuckler. and Sven Behnke, "Local Multi-Resolution Surfel Grids for MAV Motion Estimation and 3D Mapping" Proceedings of 13th International Conference on Intelligent Autonomous Systems (2014).

4. Humbert, J. S, "Bio-Inspired Visuomotor Convergence in Navigation and Flight Control Systems" $P h$. D. Dissertation, Mechanical Engineering Department, California Inst. of Technology, Pasadena, CA (2005).

5. Egelhaaf, M., Kern, R., Krapp, H. G., Kretzberg, J., Kurtz, R. and Warzecha, A. "Neural encoding of behaviourally relevant visual-motion information in the fly" Trends in Neurosciences, Vol. 25, pp. 96-102, (2002).

6. Gibson, J. The Perception of the Visual World, Houghton Mifflin Oxford, England, U.K., pp. 117-144, (1950).

7. Koenderink, J. J. and van Doorn, A. J. "Facts on Optic Flow" Biological Cybernetics, Vol. 56, pp. 247-254 (1987).

8. Conroy, J., Gremillion, G., Ranganathan, B. and Humbert, J. S. "Implementation of Wide-Field Integration of Optic Flow for Autonomous Quadrotor Navigation" Autonomous Robots, Vol. 27, pp. 189-198 (2009).

9. Humbert, J. S. and Hyslop, A. M. "Bio-Inspired Visuomotor Convergence" IEEE Transactions on Robotics, Vol. 26, pp. 121-130 (2010).

10. Hyslop, A. M., Krapp, H. G. and Humbert, J. S. "Control Theoretic Interpretation of Directional Motion 


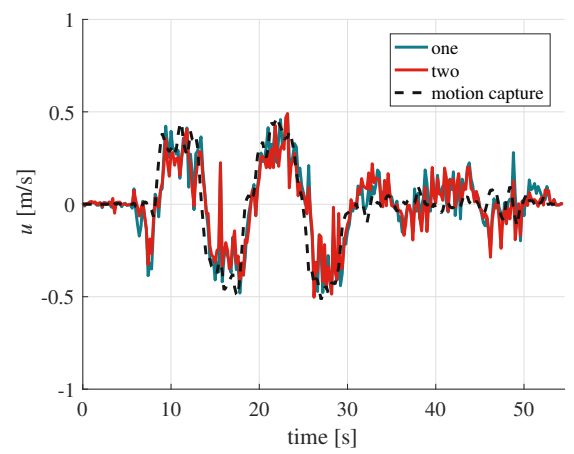

(a) Estimated velocity $u$

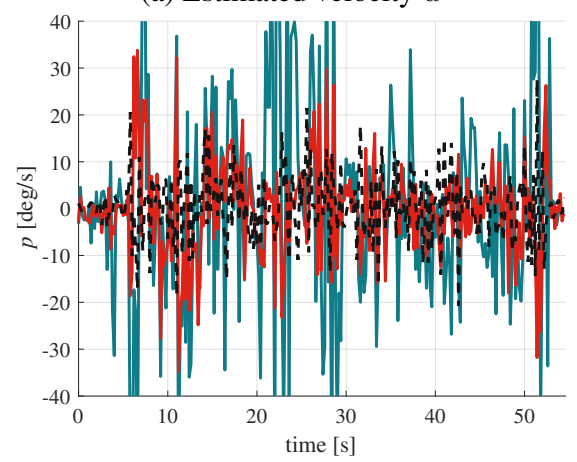

(d) Estimated angular velocity $p$

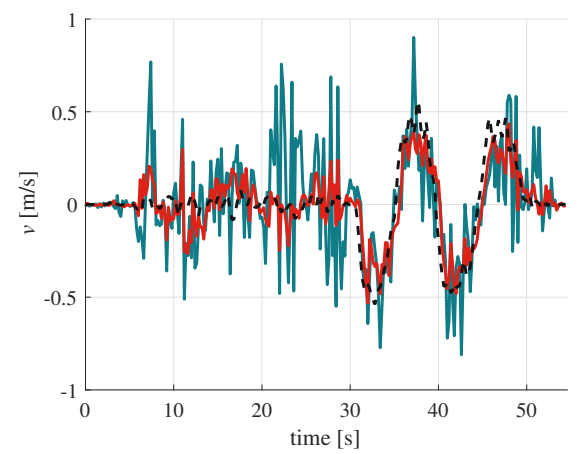

(b) Estimated velocity $v$

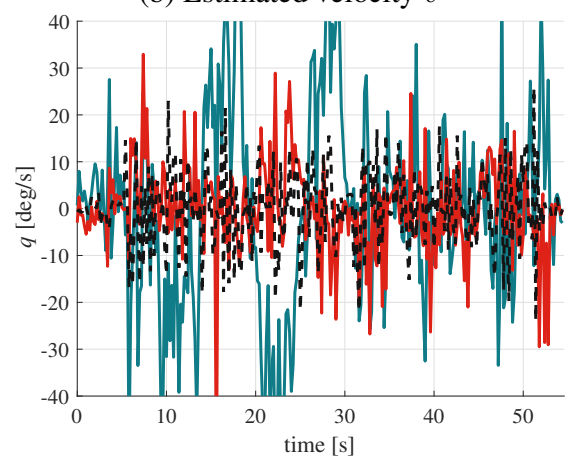

(e) Estimated angular velocity $q$

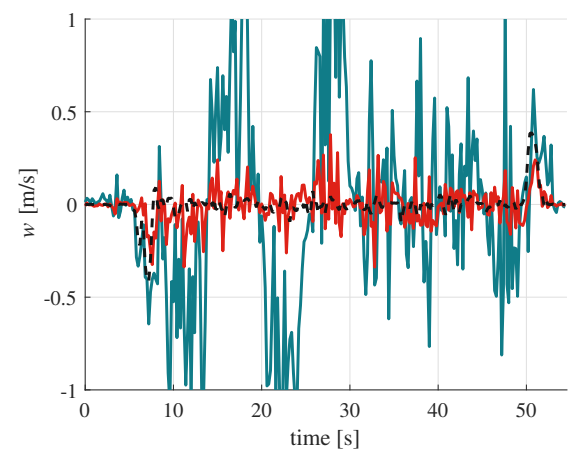

(c) Estimated velocity $w$

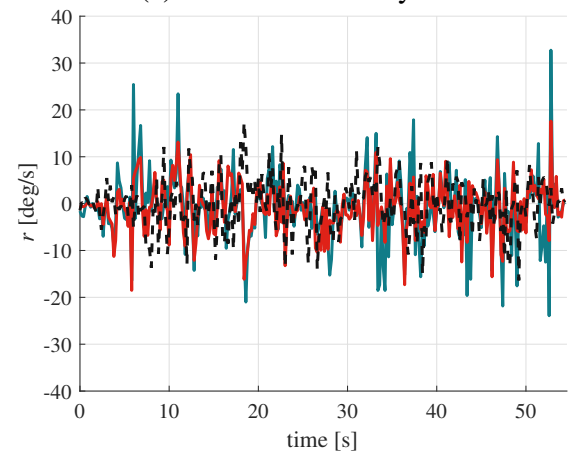

(f) Estimated angular velocity $r$

Fig. 10: Comparison of estimated motion variables for different number of cameras in experiments.

Preferences in Optic Flow Processing Interneurons" Biological Cybernetics, Vol. 103, pp. 353-364 (2010).

11. Sakamoto, H. and Hokamoto, S. "Attitude Angle Estimation of Space Probe through Wide-Field Integration of Optic Flow" Trans. of JSASS Aerospace Technology Japan, Vol. 12, ists29, pp.Pd_41-Pd_46 (2014).

12. Hyslop, A. M. and Humbert, J. S. "Autonomous Navigation in Three-Dimensional Urban Environments Using Wide-Field Integration of Optic Flow" Journal of Guidance, Control, and Dynamics, Vol. 33, pp. 147159 (2010).

13. Shoemaker, M. A. and Hokamoto, S. "Application of Wide-Field Integration of Optic Flow to Proximity Operations and Landing for Space Exploration Missions" Advances in the Astronautical Sciences, Vol. 142, pp. 23-36 (2011).

14. Kobayashi, N., Oishi, M., Kinjo, Y. and Hokamoto, S. "Experimental Verification of Wide-Field-Integration of Optic Flow for State Estimation" Trans. of JSASS Aerospace Technology Japan, Vol. 14 ists30, pp.Pd_63Pd_68 (2016).

15. Kobayashi, N., Bando, M. and Hokamoto, S. "Improvement of Wide-Field-Integration of Optic Flow Considering Practical Sensor Restrictions Journal of Mechanical Engineering and Automation, Vol. 7, No. 2, pp. 53-62 (2017).

16. Shoemaker, M. A. and Hokamoto, S. "Comparison of Integrated and Nonintegrated Wide-Field Optic Flow for Vehicle Navigation” Journal of Guidance, Control, and Dynamics, Vol. 36, pp. 710-720 (2013).

17. Hamel, T. and Samson, C. "Riccati Observers for the Non-stationary PnP Problem" IEEE Transactions on Automatic Control, Vol. 63, pp. 726-741 (2018).

18. Modenini, D. "Attitude Determination from Ellipsoid Observations: A Modified Orthogonal Procrustes Problem" Journal of Guidance, Control, and Dynamics, Vol. 41, pp. $2320-2325$ (2018).

19. Modenini, D. "Five-Degree-of-Freedom Pose Estimation from an Imaged Ellipsoid of Revolution" Journal of Spacecraft and Rockets, Vol. 56, pp. 952-958 (2019). 


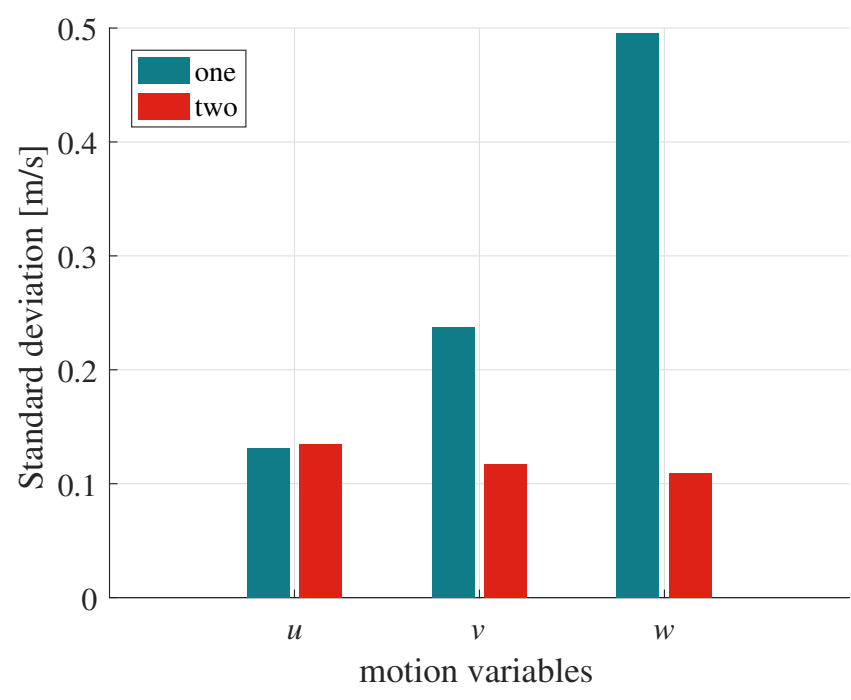

(a) Standard deviations of estimated errors in translational velocities

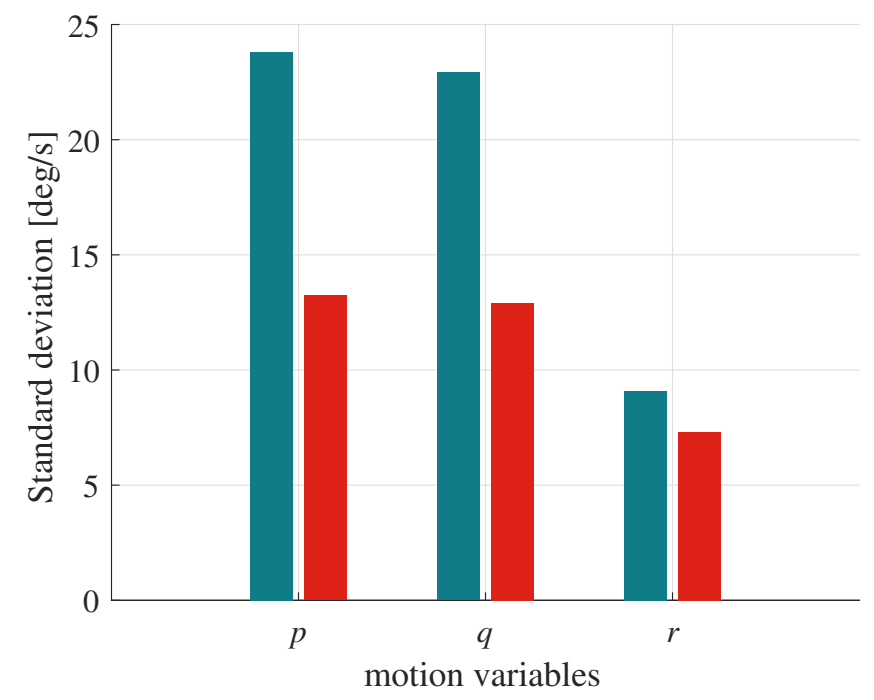

(b) Standard deviations of estimated errors in angular velocities

Fig. 11: Comparison of the standard deviations of estimated errors for each number of cameras in experiments. 